University of Nebraska - Lincoln

DigitalCommons@University of Nebraska - Lincoln

3-5-2004

\title{
Spin-Exchange-Induced Circularly Polarized Molecular Fluorescence
}

\author{
A.S. Green \\ University of Nebraska-Lincoln \\ Gordon A. Gallup \\ University of Nebraska-Lincoln, ggallup1@unl.edu \\ Mark A. Rosenberry \\ University of Nebraska-Lincoln, mrosenberry@siena.edu \\ Timothy J. Gay \\ University of Nebraska-Lincoln, tgay1@unl.edu
}

Follow this and additional works at: https://digitalcommons.unl.edu/physicsgallup

Part of the Physics Commons

Green, A.S.; Gallup, Gordon A.; Rosenberry, Mark A.; and Gay, Timothy J., "Spin-Exchange-Induced Circularly Polarized Molecular Fluorescence" (2004). Gordon Gallup Publications. 2.

https://digitalcommons.unl.edu/physicsgallup/2

This Article is brought to you for free and open access by the Research Papers in Physics and Astronomy at DigitalCommons@University of Nebraska - Lincoln. It has been accepted for inclusion in Gordon Gallup Publications by an authorized administrator of DigitalCommons@University of Nebraska - Lincoln. 


\title{
Spin-Exchange-Induced Circularly Polarized Molecular Fluorescence
}

\author{
A. S. Green, G. A. Gallup, M. A. Rosenberry, and T. J. Gay \\ Behlen Laboratory of Physics, University of Nebraska, Lincoln, Nebraska 68588-0111, USA
}

(Received 6 June 2003; published 1 March 2004)

\begin{abstract}
We have measured the circular polarization of light emitted from both atomic $\mathrm{H}$ and molecular $\mathrm{H}_{2}$ after bombarding $\mathrm{H}_{2}$ with longitudinally polarized electrons. For both atomic and molecular fluorescence near threshold we observe a circular polarization as great as $10 \%$ of the electron polarization. This represents the first direct observation of spin transfer in electron-molecule collisions.
\end{abstract}

PACS numbers: $34.80 . \mathrm{Nz}$

When spin-polarized electrons collisionally excite atoms, an incident electron may exchange with an atomic electron and give the atomic state a net spin polarization. Because of spin-orbit coupling, the subsequent fluorescence will often exhibit circular polarization. This transfer of electron spin to photon spin was first predicted qualitatively prior to 1956 by Dayhoff [1]. By 1971, several quantitative schemes to exploit it as a means of optical electron polarimetry had been discussed [2], although experimental verification of these ideas did not occur for another decade [3]. Optical electron polarimetry and the use of electron-spin-induced circularly polarized light as a probe of atomic collision dynamics are now well-developed techniques [4-8].

What might one expect to see if the atomic target is replaced with simple molecules? How would the more complicated angular momentum structure of a molecular target affect the transfer of angular momentum in these collisions? To date, three experiments have investigated collisions between polarized electrons and diatomic molecules; only one of them has studied fluorescence from excited states. All three experiments, however, indicate that exchange processes in electron-molecule collisions are qualitatively different from those in electron-atom collisions.

The Münster group of Hanne studied the differential scattering of $\sim 10 \mathrm{eV}$ electrons with polarization $P$ by $\mathrm{Na}, \mathrm{Hg}, \mathrm{O}_{2}$, and $\mathrm{NO}[9,10]$. (Mercury has a singlet ground state, $\mathrm{Na}$ and $\mathrm{NO}$ have open-shell doublet ground states, and the ground state of $\mathrm{O}_{2}$ is a triplet.) Their experiment measured the polarization $P^{\prime}$ of the outgoing electron beam scattered to angles between $0^{\circ}$ and $110^{\circ}$. The depolarization ratio, $P^{\prime} / P$, is a direct measure of electron exchange during the collision. As expected, no depolarization was found for elastic scattering from $\mathrm{Hg}$ $\left(P^{\prime} / P=1\right)$. For elastic scattering from $\mathrm{Na}$ and inelastic scattering from $\mathrm{Na}$ and $\mathrm{Hg}, P^{\prime} / P$ generally drops well below 1, indicating appreciable spin-exchange amplitudes. In contrast, $P^{\prime} / P$ does not vary significantly from unity for elastic scattering from $\mathrm{O}_{2}$ and $\mathrm{NO}$ targets with any combination of the scattering angles and energies studied, except near $100^{\circ}$ with $\mathrm{O}_{2}$ where $P^{\prime} / P$ was closer to $0.90 \pm 0.05$ over a broad range of energies. In a sepa- rate experiment, Ratliff et al. [11] determined the rate constants for spin-exchange in collisions of $\mathrm{O}_{2}$ and $\mathrm{NO}$ molecules with the thermal polarized electrons of a flowing afterglow. The values they extracted were more than an order of magnitude below those calculated for alkali atoms and $\mathrm{H}$.

These results contradict the naïve argument that for impulsive collisions molecules should look essentially like atoms to an incident electron, given that typical collision times for electrons of this energy are much shorter than any rotational or vibrational time associated with the target molecule. Theoretical calculations of elastic scattering also predict small depolarizations for these molecules, apparently because significant effects that exist for specific orientations of the internuclear axis are washed out by angular averaging [12,13]. We note, however, that theoretical calculations of electron spin depolarization during collisions with triplet excited states of $\mathrm{H}_{2}$ molecules find large average depolarization effects [14].

More recently, the Münster group did an electron/ photon spin-transfer experiment of the type mentioned earlier [15]. They bombarded $\mathrm{N}_{2}$ in its singlet closed-shell ground state with beams of $14.5 \mathrm{eV}$ polarized electrons and measured the circular polarization, $P_{3}$, of the resultant $\mathrm{C}^{3} \prod_{u}-\mathrm{B}^{3} \prod_{g} 337 \mathrm{~nm}$ light. Fluorescence experiments are quite different from those where the outgoing electron is observed, because the long times required for the excited state to decay allow the rotational and spin degrees of freedom of the molecule to couple to the electron spin. Within their statistical uncertainty of $2 \times$ $10^{-3}$ the Münster group found $P_{3}$ to be nil. In contrast, experiments with noble gas targets, in which triplet states are produced by pure exchange excitation, result in large values of $P_{3}[6,8]$.

This Letter reports on an experiment we have done with molecular hydrogen to further investigate these issues. We have observed, apparently for the first time, circularly polarized fluorescence from a molecular target induced by exchange with a beam of spin-polarized electrons [16]. This constitutes the first direct evidence for spin polarization of the target in electron-molecule collisions. Interestingly, we observe significant values of $P_{3}$ for two processes: the simple excitation of molecular 
hydrogen excited states and the dissociative excitation of atomic hydrogen.

Our system, shown schematically in Fig. 1, uses a GaAs crystal as the source of polarized electrons. Photoemission is induced by a $50 \mathrm{~mW} 785 \mathrm{~nm}$ laser diode module; a Pockels cell (PC) is used to set the photon helicity. We typically obtain an electron beam of 6-12 $\mu \mathrm{A}$ with $26 \%$ longitudinal polarization, $P_{e}$, and an energy width of $<0.4 \mathrm{eV}$. The electrons are steered to a target chamber where they collide with the hydrogen molecules. (Since we have a flowing gas system, the equilibrium fraction of atomic $\mathrm{H}$ is negligible.) We find no significant dependence of $P_{3}$ on the $\mathrm{H}_{2}$ pressure between 4 and 18 mTorr, so we used 12 mTorr for most of the data acquisition. By varying the voltage of the target chamber, we change the electron's incident energy from 0 to $110 \mathrm{eV}$. We observe the fluorescence emitted in the forward direction using an in-line optical polarimeter [17]. The scattered electrons are not detected. A uniform magnetic field of $10^{-2} \mathrm{~T}$ is applied parallel to the electron beam axis and is required to guide the beam and reduce the number of scattered electrons which reflect back into the target chamber.

To acquire data, the PC is modulated between helicity states, and the corresponding variation in the photon count rate is used to generate an asymmetry. By rotating the quarter-wave plate contained within the optical polarimeter we can determine how much of this asymmetry is due to spurious systematic effects (e.g., helicitydependent intensity variations) and how much to a real circular polarization $P_{3}$. As a systematic check, we have confirmed that no $P_{3}$ is measured when the PC is set to produce linearly polarized light.

The electric potential of the system (referenced to the GaAs crystal) before and after the target region is kept below $15 \mathrm{~V}$ to ensure that no fluorescence occurs in these regions. In principle this configuration leads to "trapped" electrons which could scatter multiple times at progressively lower energies. However, we have also taken data with other voltage configurations and obtain similar results for both the optical excitation functions and $P_{3}$.

The bandpass filter within the optical polarimeter was varied to look at different parts of the Fulcher band fluorescence spectrum. All filters eliminated counts from stray laser or room light. For the molecular signal, we used filters for light at $600 \mathrm{~nm}(10 \mathrm{~nm}$ FWHM) and $588.3 \mathrm{~nm}(0.3 \mathrm{~nm}$ FWHM). The region near $600 \mathrm{~nm}$

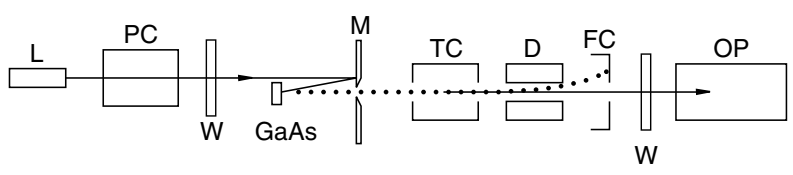

FIG. 1. Apparatus schematic showing laser (L), Pockels cell (PC), vacuum windows (W), GaAs crystal, mirrored aperture (M), target chamber (TC), deflector plates (D), Faraday cup (FC), and the optical polarimeter (OP). has a number of strong lines from four different excited triplet states $\left(3 d \sigma^{3} \Sigma_{g}-2 p \pi^{3} \Pi_{u}, 3 d \pi^{3} \Pi_{g}-2 p \pi^{3} \Pi_{u}\right.$, $3 s \sigma^{3} \Sigma_{g}-2 p \pi^{3} \Pi_{u}$, and $3 p \pi^{3} \Pi_{u}-2 s \sigma^{3} \Sigma_{g}$ ) and very few associated with singlet states [18]; assuming equal excitation probabilities, about $94 \%$ of the observed light should come from triplet levels. The $588.3 \mathrm{~nm}$ filter was used to isolate a specific molecular transition: $3 d \pi^{3} \Pi_{g}(\nu=1)-2 p \pi^{3} \Pi_{u}(\nu=1)$. This transition accounted for roughly $80 \%$ of the light passed by the filter, with the remaining $20 \%$ coming from a single doubly excited state. The electronic states associated with these transitions are shown in Fig. 2.

When looking for the atomic signal, we used a filter centered at $656 \mathrm{~nm}(\mathrm{H} \alpha)$, allowing us to observe molecules which dissociate with at least one atom in the $n=3$ state. These dissociating states have the same asymptotic energy as the molecular triplet states above; in some cases a given molecular state will have a finite probability for both dissociation and fluorescence (see Fig. 2). Most of our atomic data were acquired with an $11 \mathrm{~nm}$ FWHM filter, but we later confirmed these data using a narrower $1.5 \mathrm{~nm}$ FWHM unit, indicating that molecular fluorescence contributions are small at this wavelength.

Figure 3 displays our optical excitation functions for all of the above cases. For comparison, we have also included the $\mathrm{H} \alpha$ data of Vroom and de Heer [19]. The tuning of the electron beam can affect the measured energy scale by several volts, although for the same tuning all of the filters give consistent threshold energies. The horizontal scale of the figure is set so that the atomic signal threshold is close to the theoretical value of $16.6 \mathrm{eV}$. As a result of this adjustment, we find that the molecular signal threshold is near $14 \mathrm{eV}$, close to the predicted value of $13.9 \mathrm{eV}$. Tuning also had a modest effect on the efficiency of light collection, so the atomic curves were set to match at $45 \mathrm{eV}$. The shapes of the excitation functions for our $\mathrm{H} \alpha$ curves are in qualitative agreement with each other and the results of [19]. The molecular excitation functions are

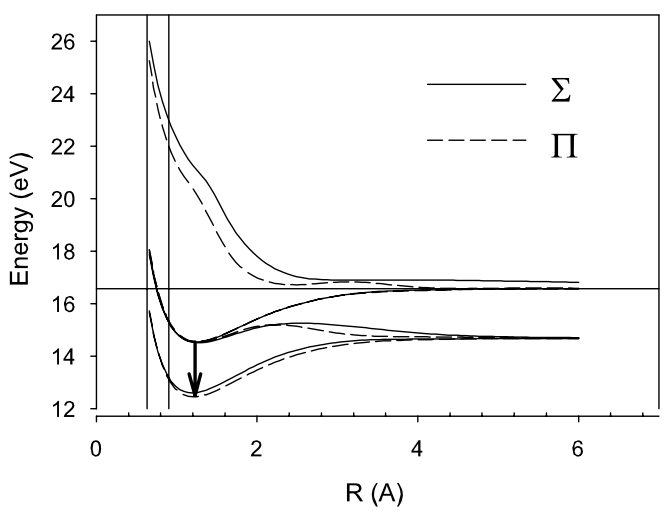

FIG. 2. Potential energy curves of $\mathrm{H}_{2}$ triplet states leading to $\mathrm{H}(n=2,3)$ states. The Frank-Condon region lies between the vertical lines; the horizontal line is the threshold for $\mathrm{H}(1 s)+$ $\mathrm{H}(3 s)$ : $16.57 \mathrm{eV}$. The vertical arrow indicates the molecular transitions we observe. 


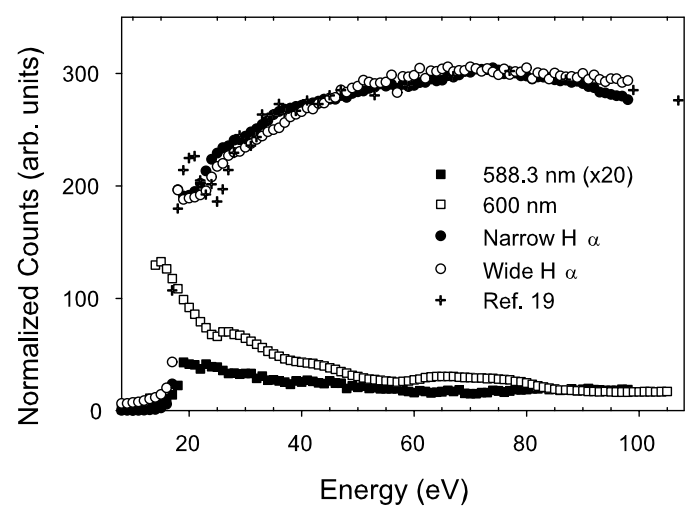

FIG. 3. Excitation curves for atomic and molecular fluorescence normalized to pressure and beam current. Signals obtained with the $588.3 \mathrm{~nm}$ filter are multiplied by 20 to be visible on this scale.

much more sharply peaked, presumably because there are no ionization channels into which the incident electron can dump energy.

Figure 4 displays the measured $P_{3}$ values for all the transitions studied. The molecular polarizations observed with the $600 \mathrm{~nm}$ filter are significantly higher than those of $\mathrm{H} \alpha$. Surprisingly, $P_{3}$ for the ${ }^{3} \Pi_{g^{-}}{ }^{3} \Pi_{u}$ light passed by the $588.3 \mathrm{~nm}$ filter is smaller than that for both the mix of molecular transitions (primarily ${ }^{3} \Sigma-{ }^{3} \Pi$ and ${ }^{3} \Pi-{ }^{3} \Sigma$ ) passed by the $600 \mathrm{~nm}$ filter and the atomic signal. In the case of $\Sigma-\Pi$ or $\Pi-\Sigma$ transitions, the fluorescence results from dipole transitions perpendicular to the internuclear axis, while in the $\Pi-\Pi$ case, it results from parallel transitions. This difference might cause $P_{3}$ values to differ quantitatively.

The slow decay of $P_{3}$ with incident electron energy is also of interest: it remains nonzero even $50 \mathrm{~V}$ above the excitation threshold. This is quite different from electron excitation of the alkali atoms, where $P_{3}$ falls to zero by $10 \mathrm{~V}$ above threshold [20]. However, a long tail is characteristic of the pure exchange excitation of noble gas atoms by polarized electrons [6]. Since the $\mathrm{H}_{2}$ molecule is a closed-shell system, it seems reasonable that the exci-

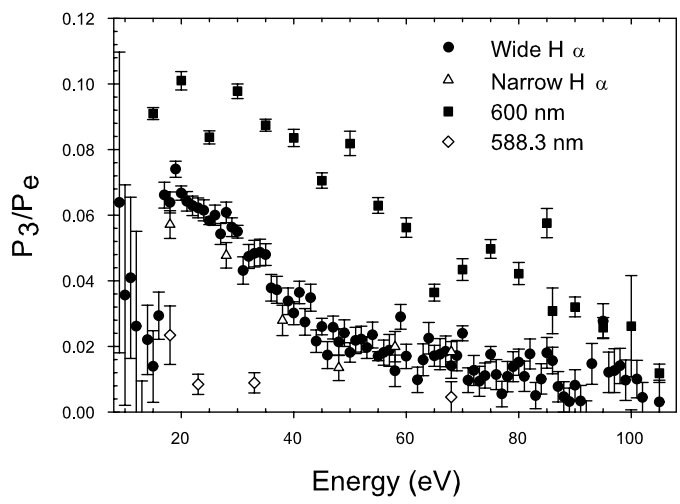

FIG. 4. $P_{3} / P_{e}$ versus incident electron energy for molecular and atomic fluorescence. tation process would resemble the noble gases more than the alkalis, even if the molecule subsequently dissociates.

The longitudinal $B$ field in the target region should not alter the observed $P_{3}$ values significantly. Indeed, it eliminates potential problems related to Hanle depolarization [21]. A potential complication is depolarization due to Paschen-Back (PB) decoupling of $L$ and $S$ in the excited atomic or molecular states. This will be small for our field, which is at most $13 \%$ of the PB field parameter $\mu_{B} B / \Delta E_{F S}$, where $\Delta E_{F S}$ is the $L$-dependent fine-structure splitting of the atomic or molecular excited states [22]. Our $P_{3}$ values thus represent a lower limit on field-free values of $P_{3}$. Measurements of $P_{3}$ with $\mathrm{Ar}$ targets made to determine $P_{e}$ gave no indication of $\mathrm{PB}$ depolarization.

When thinking about the dynamics of spin transfer, it is useful to keep in mind the relevant time scales. The incident electron is moving fast enough that the molecule is essentially fixed during the collision $\left(10^{-16} \mathrm{sec}\right)$. For these conditions, it is very likely that the nuclei will remain in the Frank-Condon region (internuclear separation of 0.6 to $0.9 \AA$ [23], see Fig. 2) as it climbs to an excited triplet state. If this state has enough energy to dissociate, it does so on the order of $10^{-14} \mathrm{sec}$, comparable to the classical vibrational period and faster than the rotation period of $10^{-13}$ seconds. In contrast, the length of time needed to transfer the polarized electron spin into oriented orbital angular momentum is much longer, around $10^{-9} \mathrm{sec}$, and the fluorescence occurs even later, at $10^{-8} \mathrm{~s}$. From this it is clear that the initial insertion of the electron into a triplet state will be the same for both the atomic and molecular signals. However, dissociation of the molecule takes place very rapidly compared to the coupling times, so the molecular and the atomic cases evolve separately.

To compare our results to those for known atomic data, we consider the "spin transfer efficiency" $T$, equal to the initial spin polarization of the excited system (before any fine or hyperfine depolarization [24]) divided by $P_{e}$. If we excite a pure molecular triplet state by exchange, $T=2 / 3$ [25]. We now make the simplifying assumptions that following breakup, the $3 s, 3 p$, and $3 d$ states have the same electronic polarization $T^{\prime}$, and that there is complete fine-structure depolarization of the $3 p$ and $3 d$ states, with no coupling of electron spin to the nuclei due to the ambient $B$ field. We also account for the branching ratio between $\mathrm{H} \alpha$ and $\mathrm{Ly} \beta$ radiation from the $3 p$ state, and assume that light from the $3 s$ state is unpolarized. Given our measured $P_{3} / P_{e}$ ratio of 0.065 near threshold, and taking the relative initial populations of the three sublevels as those given for electron impact excitation of $\mathrm{H}$ [26], we obtain $T^{\prime}=0.37$. Assuming equal initial populations of the three substates, $T^{\prime}=0.47$. This indicates that polarization losses prior to and during dissociation are modest. It also compares well with experimental values of $T$ for the alkali atoms from $\mathrm{Na}$ $(T \sim 0.25)$ to $\mathrm{Cs}(T \sim 0.45)[20]$. 
The molecular fluorescence data can be contrasted with data from an isoelectronic atom, i.e., He. Excitation of the $(1 s 3 p)^{3} P$ state of He gives a threshold value of $P_{3} / P_{e}=$ 0.5 [17]. However, $P_{3} / P_{e}$ for our $600 \mathrm{~nm}$ molecular fluorescence is only 0.1 at threshold, despite having the same $T=2 / 3$. From this, we can see that the evolution of the initial spin angular momentum is quite different for the two cases.

Spin coupling in the molecular case is complicated because of the rotation of the internuclear axis. If the spinorbit coupling is quite strong and the molecular rotation is fairly slow, the electron spin will be able to follow the changing magnetic field in the molecule. This means that the electron spin will become locked to the molecule's (rotating) axis, and averaging will result in a zero $P_{3}$. On the other hand, if the spin-orbit coupling is weaker and the molecule is rotating rapidly, then the electron spin will be unable to follow the rapidly changing magnetic field of the molecule, and it will remain oriented in the laboratory frame. In this case a significant $P_{3}$ may be observed for the molecular fluorescence.

The case of strong coupling and low rotation is designated Hund's Case (a); weaker coupling and high rotation is Hund's Case (b). The transition from one regime to the other is set by the spin uncoupling constant $Y=A / B$, where $A$ (the spin-orbit coupling constant) determines the strength of the interaction between the electron spin and the molecular magnetic field, and $B$ is the rotational constant of the molecule (proportional to the molecule's angular velocity) [27]. The transition point between the two cases occurs when $J \gg 2 \sqrt{Y}$. These considerations explain why we measure significant values of $P_{3}$ for $\mathrm{H}_{2}$ while the one $N_{2}$ transition studied yields $P_{3}$ values consistent with zero [15]. For a typical ${ }^{3} \Pi$ state of $\mathrm{H}_{2}$, $Y=0.003$, and the spin decouples for any nonzero $J$. In contrast, the $\mathrm{C}^{3} \Pi_{u}$ state of $N_{2}$ has $Y=25$, and the spin does not decouple until $J \gg 10$ [28]. Thus at room temperature most of the populated $\mathrm{H}_{2}$ states will maintain their polarization long enough to affect the fluorescence. Conversely, most of the $\Pi$ states of $N_{2}$ have their spin strongly coupled to the molecular rotation, and they will lose their polarized character.

In conclusion, we have observed circularly polarized fluorescence from both $\mathrm{H}_{2}$ molecules and $\mathrm{H}$ atoms, demonstrating spin-transfer effects in polarized electron- $\mathrm{H}_{2}$ collisions. This technique represents a useful new probe of exchange mechanisms in electron-molecule scattering, and opens a new window on spin-orbit coupling dynamics in molecules.

We wish to thank Paul Burrow and Tom Rescigno for useful discussions. This work was supported by NSF Grant No. PHY-0099363.

[1] E. S. Dayhoff (private communication), as quoted by H. A. Tolhoek, Rev. Mod. Phys. 28, 277 (1956).
[2] P. S. Farago and J. S. Wykes, J. Phys. B 2, 747 (1969); J. S. Wykes, ibid. 4, L91 (1971).

[3] M. Eminyan and G. Lampel, Phys. Rev. Lett. 45, 1171 (1980).

[4] A. Wolcke, K. Bartschat, K. Blum, H. Borgmann, G. F. Hanne, and J. Kessler, J. Phys. B 16, 639 (1983).

[5] K. Bartschat, U. Thumm, and D.W. Norcross, J. Phys. B 25, L641 (1992).

[6] J. E. Furst, W. M. K. P. Wijayaratna, D. H. Madison, and T. J. Gay, Phys. Rev. A 47, 3775 (1993).

[7] R Srivastava, R. P. McEachran, and A. D. Stauffer, J. Phys. B 28, 869 (1995).

[8] D. H. Yu, P. A. Hayes, J. E. Furst, and J. F. Williams, Phys. Rev. Lett. 78, 2724 (1997).

[9] T. Hegemann, M. Oberste-Vorth, R. Vogts, and G. F. Hanne, Phys. Rev. Lett. 66, 2968 (1991).

[10] T. Hegemann, S. Schroll, and G. F. Hanne, J. Phys. B 26, 4607 (1993).

[11] J. M. Ratliff, G. H. Rutherford, F. B. Dunning, and G. K. Walters, Phys. Rev. A 39, 5584 (1989).

[12] F. J. da Paixão, M. A. P. Lima, and V. McKoy, Phys. Rev. Lett. 68, 1698 (1992); Phys. Rev. A 53, 1400 (1996).

[13] G. Woste, K. Higgins, P. Duddy, C. M. Fullterton, and D. G. Thompson, J. Phys. B 29, 2553 (1996).

[14] C. S. Sartori, FJ. da Paixao, and M. A. P. Lima, Phys. Rev. A 55, 3243 (1997).

[15] G. F. Hanne, in Novel Aspects of Electron-Molecule Collisions, edited by K. Becker (World Scientific, Singapore, 1998); C. Mette et al., Verh. Dtsch. Phys. Ges. (VI) 29, 462 (1994).

[16] Although not directly related to this work, we note that nonzero values of $P_{3}$ have been observed for $\mathrm{H}_{2}$ molecular fluorescence in unpolarized electron-photon coincidence measurements: M. A. Khakoo and J.W. McConkey, J. Phys. B 20, L175 (1987); J.W. McConkey, S. Trajmar, J. C. Nickel, and G. Csanak, J. Phys. B 19, 2377 (1986).

[17] T. J. Gay, J. E. Furst, K.W. Trantham, and W. M. K. P. Wijayaratna, Phys. Rev. A 53, 1623 (1996).

[18] H. M. Crosswhite, The Hydrogen Molecule Wavelength Tables of Gerhard Heinrich Dieke (Wiley-Interscience, New York, 1972).

[19] D. A. Vroom and F. J. de Heer, J. Chem. Phys. 50, 580 (1969).

[20] C. P. $\mathrm{Na} \beta$, M. Eller, N. Ludwig, E. Reichert, and M. Webersinke, Z. Phys. D 11, 71 (1989).

[21] K.W. Trantham, T. J. Gay, and R. J. Vandiver, Rev. Sci. Instrum. 67, 4103 (1996).

[22] H. A. Bethe and E. E. Salpeter, Quantum Mechanics of One- and Two-Electron Atoms (Springer, Berlin, 1957).

[23] T. E. Sharp, Atomic Data 2, 119 (1971).

[24] K. Blum and H. Jakubowicz, J. Phys. B 11, 909 (1978).

[25] B. K. Blum, Density Matrix Theory and Applications (Plenum, New York, 1996), 2nd ed., p. 133.

[26] W. Kedzierski, A. Abdellatif, J.W. McConkey, K. Bartschat, D. V. Fursa, and I. Bray, J. Phys. B 34, 3367 (2001).

[27] G. Herzberg, Molecular Spectra and Molecular Structure I. Spectra of Diatomic Molecules (Van Nostrand and Reinhold Co., New York, 1950), 2nd ed.

[28] K. P. Huber and G. Herzberg, Molecular Spectra and Molecular Structure IV: Constants of Diatomic Molecules (Van Nostrand and Reinhold Co., New York, 1979). 